
Оригинальная статья / Original article
https://doi.org/10.18619/2072-9146-2019-5-94-97
УДК 005.57

Шабанов Т.Ю.

000 «ТТП»

454021, Россия, Челябинск, а/я 18560 СтП

E-mail shabanovtyu@mail.ru

Конфликт интересов: Автор заявляе об отсутствии конфликта интересов.

Для цитирования: Шабанов Т.Ю Время сделки: оптимизация лага. Овощи России. 2019;(5):94-97.

https://doi.org/10.18619/2072-9146-2019-5-94-97

Поступила в редакцию: 05.06.2019

Принята к печати: 26.06.2019

Опубликована: 25.10.2019

Timofey Yu. Shabanov

Company STP

PO 18560 STP, Chelyabinsk, Russia, 454021

E-mail: shabanovtyu@mail.ru

Conflict of interest: author declare no conflict of interest.

For citation: Shabanov T.Yu. Time deals: optimization of lag. Vegetable crops of Russia. 2019;(5):94-97 (In Russ.)

https://doi.org/10.18619/2072-9146-2019-5-94-97

Received: 05.06.2019

Accepted for publication: 26.06 .2019

Accepted: 25.10 .2019

\section{Время сделки: оптимизация лага}

\section{PE3ЮME}

Актуальность. Во многом успешная сделка купли-продажи определяется временем совершения. Поиск наилучшего времени сделки увязан с оптимизацией временного лага (от англ. lag - запаздывание). Цель исследования - оптимизация временного лага аграрного рынка.

Материалы и методы. В статье рассмотрена роль времени в бизнес-процессах на примере рыночного предложения картофеля за период 1998-2018 годов.

Результаты. Выявлена тенденция ежегодного сокращения на 365 тыс. т $(1,2 . .1,7 \%$ от общего количества) предложения картофеля отечественным производителем. Выявлен приоритет количества предложения картофеля перед ценой производителя для сделок на аграрном рынке РФ. Показан обратнопропорциональный характер зависимости цены производителя от количества картофеля, т.е. чем хуже урожайность, тем выше цена производителя. Сокращение предложения картофеля на 1 млн т на аграрном рынке РФ может привести к росту цены производителя на 8,5 \$/т (4-5\% в среднем). Изложена концепция и подтверждена гипотеза о линеаризации равновесных рыночных процессов в условиях временных сдвигов динамики их параметров. Проверено предположение о существование межинтервального временного оптимума, при котором линеаризация бизнес-процессов максимальна. В ходе корреляционного анализа динамики годовых показателей рынка картофеля выявлено, что месяц май текущего периода - оптимальное время предложений производителя при ограничениях цены и убывающей доходности, и месяц сентябрь - при ограничениях количества и возрастании доходности. При государственном фиксировании цены производителя на агарном рынке сделки более вероятны в мае, при неурожае (ограниченном количестве) - в сентябре.

Ключевые слова: экономика, аграрный рынок, рынок картофеля, методология, равновесие, линеаризация, оптимизация, временной лаг, время сделки.

\section{Time deals: optimization of lag}

\section{ABSTRACT}

Relevance. IIn many ways, a successful purchase and sale transaction is determined by the time it takes to complete it. The search for the best deal time is linked to the optimization of the time lag. The purpose of the study is to optimize the time lag of the agricultural market.

Methods. This article considered a role of time in business processes for Russian potato market of producer into period 1998-2018.

Results. There was trend of annual reduction about 365 thousand tons (1.2...1.7\% of total) per year supply of potatoes on Russian domestic market of producers. Some priority of quantity vs price of producer been revealed. There was inverse proportional function between producers' prices and quantity of potatoes. That is than worse harvest that higher producer's price. Reducing a supply of potatoes 1 million tons from agricultural market perhaps increase in producer prices by $\$ 8.5 / \mathrm{t}$ (4...5\% on average). Offered concept and hypothesis about linearization of equilibrium of market processes into conditions of time shifts of dynamics their parameters were confirmed. There verified assumption about existence of inter-interval time optimum which this linearization maximal business processes. In course of correlation analysis of dynamics of annual indicators of potato market, it revealed month - May of current period as optimal time of producer's offers with price restrictions and diminishing returns, and month - September with quantity restrictions and increasing returns. If fixation of producer's prices situation of deals - May more likely, in case of crop failure (limited quantity) in September.

Keywords: economy, agricultural market, potato market, methodology, balance, linearization, optimization, time lag, time of deals. 


\section{Введение}

о многом успешная сделка купли-продажи опреде-

ляется временем совершения. Поиск наилучшего времени сделки увязан с оптимизацией временного лага (от англ. lag запаздывание). Монографический анализ научных работ [1-4] показывает актуальность вопроса, однако изложение существующих решений затруднительно для практического применения.

Предложенная концепция циклического временного лага (рис.1) основана на допущении о существовании такого лага $\Delta$ цены/количества продукта, при котором линеаризация (от лат. linearis - линейный) зависимости цены/количества формирует рыночное равновесие спроса-предложения. В этой связи, оптимизация временного лага - поиск такого отставания $-\Delta$ или опережение $+\Delta$ во времени Т цена/количество $\mathrm{Y} / \mathrm{X}$, при котором достигается наилучшая линеаризация их зависимости. В качестве критерия линеаризации выбран известный коэффициент корреляции Пирсона R (рис.1). Положительный $+\mathrm{R}$ демонстрирует прямую пропорциональную зависимость, а отрицательный - обратную. При этом степень линеаризации тем выше, чем ближе показатель единичному значению. Таким образом, поиск решения трансформируется в задачу корреляционного анализа.

На рис. 1 показана связь линеаризации $\mathrm{Y}$ от $\mathrm{X}$ при наличии временного лага для $X$. Очевидно существование такого лага $\Delta$, при котором корреляционная зависимость $Y$ от X линеаризуется.

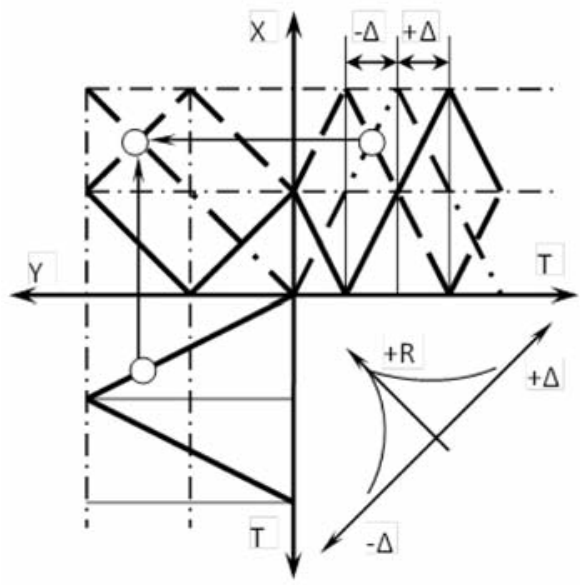

Рис. 1. Концепция и метод циклического временного лага Fig. 1. The concept and method of cyclic time lag

Цель исследования - оптимизация временного лага аграрного рынка.

Для достижения данной цели, последовательно были решены следующие задачи:

Сформированы концепция и методика оптимизации циклического временного лага;

Апробирована методика для рынка картофеля РФ.

\section{Методы}

Особенности статистических показателей, как комплексность и дискретность, затрудняют использование данных цены и количества продукта в построении кривых спроса-предложения рыночного механизма. Сложность в том, что статистические данные являются дискретным результатом рыночной конъюнктуры и комплексно характеризуют состояния за годовой временной интервал. Другими словами, сложность в определении месячных значений годового интервала. Используем для решения этого вопроса следующий подход (рис.2).

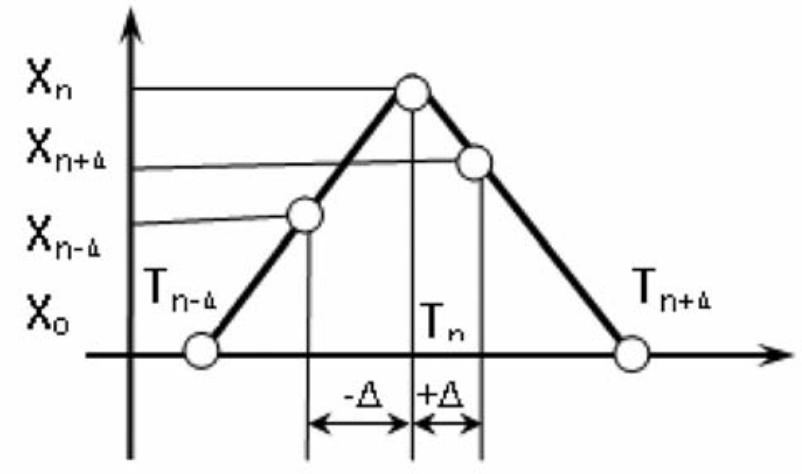

Рис.2. Определение значений временного лага Fig. 2. Definition of time lag values

В определении месячных значений временного лага $\Delta$ лежит предположение о линейном и непрерывном формировании годовых значений Т. Таким образом, вопрос определения месячных значений временных лагов сводится к задаче регрессионного анализа, т.е. поиску решения уравнения вида (1) по расчетным значениям (рис.2):

$$
\mathrm{X} \Delta=\frac{\mathrm{Xn}-\mathrm{X} 0}{\mathrm{Tn}-\mathrm{T} 0} \times \Delta \mathrm{T}+\mathrm{X} 0
$$

Разбиваем годовой период на равные месячные интервалы, их значения для цены/количества. Зная расчетные значения цены/количества продукта для месячных лагов, проведем корреляционный анализ, выявим зависимость коэффициента корреляции Пирсона для значений цены/количества продукта для каждого из месячных лагов. Оптимум этой зависимости определит оптимальный период линеаризации, т.е. значение временного лага как время сделки на аграрном рынке.

\section{Результаты}

В качестве исходных данных воспользуемся статистическими регистрами Федеральной службы государственной статистики (см. - http://www.gks.ru) - валовые сборы сельскохозяйственных культур по Российской Федерации, хозяйства всех категорий для картофеля, тыс. т; средние цены на картофель производителей сельскохозяйственной продукции по Российской Федерации в 1998-2018 годах, в среднем в год в рублях

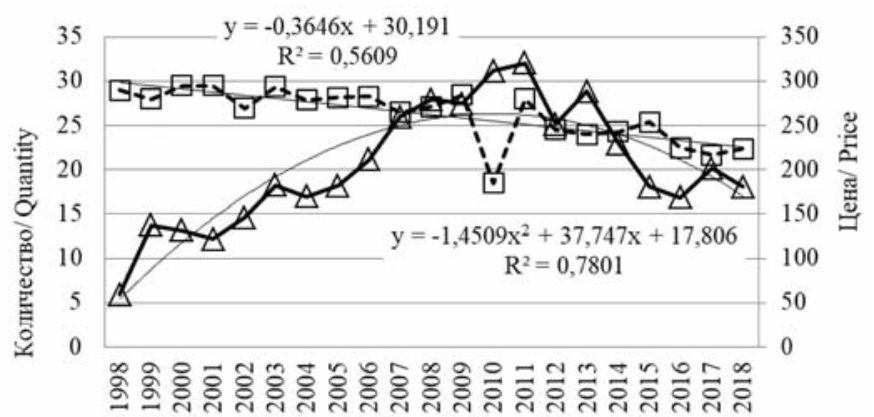

\footnotetext{
--2-Количество картофеля, млн.т Quantity of potatoes, mln. Tons

——Цена картофеля, усл.ед./т Potato price \$ / T
}

Рис.3. Динамика цены и количества картофеля за период 1998-2018 годов

Fig. 3. The dynamics of the price and quantity of potatoes for the period 1998-2018 
за тонну. Для компенсации инфляционной погрешности используем открытые данные Центрального Банка РФ (см. - http://www.cbr.ru) о курсе доллара США в рублях РФ на конец года. Проведем перерасчет рублевых цен в условные единицы (доллары США). Полученные результаты изложим графически (рис. 3).

Заметим, что использование валового сбора картофеля для оценки количества продукта на рынке объясняется особенностью хранения и реализации этой культуры производителем. Несмотря на разовый одноили двукратный ежегодный сбор урожая в Российской Федерации, реализация картофеля производителем может проводиться круглогодично из овощехранилищ. Вместе с тем, срок хранения собранной культуры редко превышает годовой период.

C помощью компьютерной программы MS Excel подберем форму кривых трендов и их зависимостей в направлении максимизации величины коэффициента достоверности $\mathrm{R}^{2}$. Коэффициент принимает значения от 0 до 1 ед. Чем ближе величина коэффициента к 1 ед., тем сильнее зависимость. При оценке полученных результатов это интерпретируется как соответствие модели данным. Для приемлемых моделей коэффициент должен быть не меньше 0,5 ед. Значение коэффициента, соответствующее 1 ед., означает функциональную зависимость между переменными.

Анализ динамики показателей (рис.3) выявляет ежегодное сокращение валового сбора картофеля в среднем на 364,6 тыс.т, что составляет 1-2\% от общего количества. Следует отметить значительные колебания цены картофеля, связанные с изменениями в агротехнике возделывания культуры, урожайностью и размерами посевных площадей.

На основании ранее изложенной методики (рис. 1, 2 и формулы 1), данных (рис.3), рассчитываемых значениях количества картофеля для континуума (от лат. continuum - непрерывное, сплошное) месячных лагов при ценовой константе (лат. constanta - постоянная, неизменная), определим коэффициент корреляции (рис. 4).

Поясним полученные значения (рис 4). Временной лаг $\Delta=0$ соответствует значениям на 31 декабря текущего периода, значения $\Delta=+1$ соответствует дате 31 января будущего года, а $\Delta=-1-31$ ноября текущего периода. Таким образом, при временном лаге $\Delta=-1$ для континуума количества и ценовой константе сопоставляются значения количества на 31 ноября и цены на 31 декабря текущего периода. При $\Delta=+1$ для континуума количества и ценовой константе сопоставляются значения количества на 31 января будущего периода и цены на 31 декабря текущего периода. Таким образом, опреде-

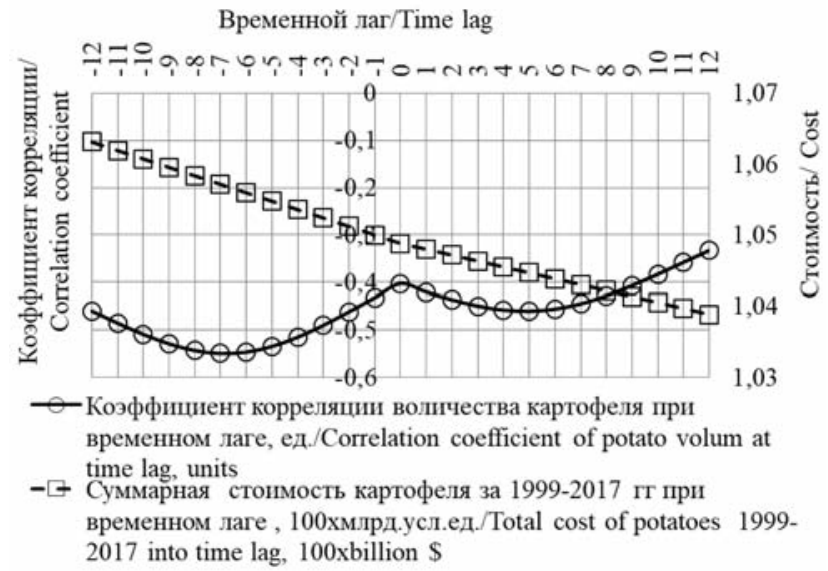

Рис.4. Корреляционный анализ количества

картофеля за период 1999-2017 годов

Fig. 4. Correlation analysis of the number

of potatoes for the period 1999-2017 ляются значения количества и цены продукта для месячных лагов, рассчитываются значения коэффициента корреляции (рис.4). Для дополнительного контроля суммируются годовые стоимости картофеля за девятилетний период 1999-2017 годов для каждого месячного лага и отображаются в виде динамики (рис.4). Анализ расчетных данных (рис.4) для континуума количества и ценовой константе рынка картофеля выявляют экстремумы (оптимальное время сделки) коэффициента корреляции (наилучшую линеаризацию обратной регрессии) цены/количества) на 31 мая ( $\Delta=-7)$ текущего периода и май $(\Delta=+5)$ будущего периода. При этом суммарная стоимость картофеля за период тем меньше, чем больше задержка сделки. Таким образом, при фиксированных ценах для производителей картофеля оптимальное время сделки для количества картофеля 31 мая при цене на 31 декабря текущего периода (рис.5). Цена текущего года на картофель может формироваться в мае. Если цена будет фиксирована на период, то более раннее совершение сделки формирует большую стоимость (выручку) реализованного картофеля. При этом,

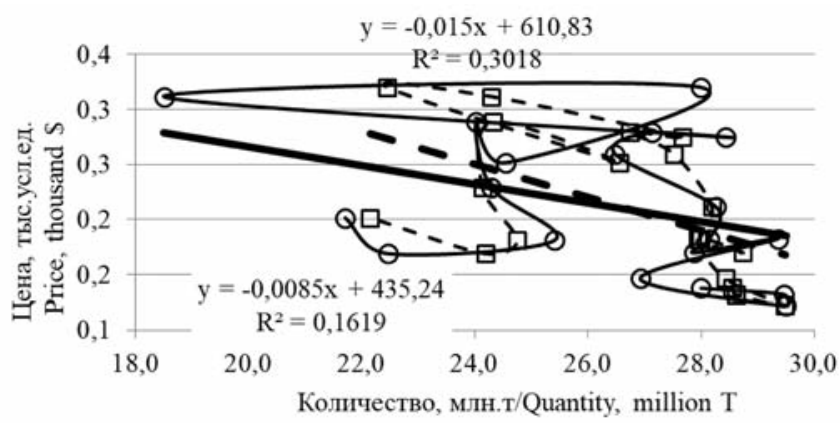

๑-Динамика картофеля факт. за период 1999-2017 гг./ Dynamics of potato fact. 1999-2017

-๘ Динамика картофеля для количества при $\Delta=-7$ п факт.цене/Potato dynamics of quantity at $\Delta=-7$ and fact.price

\section{Рис.5. Сопоставление расчетного}

и фактического количества при

фиксированной цене на картофель (1999-2017 годы)

Fig. 5. Comparison of estimated and actual

quantities at a fixed price for potatoes (1999-2017)

чем больше количество картофеля на рынке, тем меньше цена по сделке.

Рассмотрим динамику количества (на 31 декабря) к цене (на 31 декабря) картофеля (рис.5). При фактических данных имеет место линейная зависимость $\mathrm{y}=$ $0,0085 x+435.24$ при коэффициенте достоверности $\mathrm{R}^{2}=0.1619$. Значение коэффициента достоверности 0,1619 указывает на отсутствие явно выраженной линей-

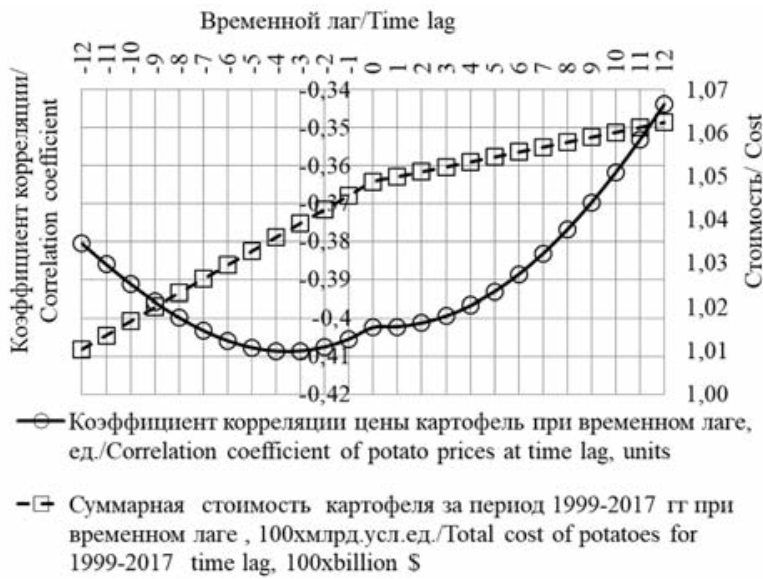

Рис.6. Корреляционный анализ цены картофеля

за период 1999-2017 годов

Fig. 6. Correlation analysis of the price

of potatoes for the period 1999-2017 


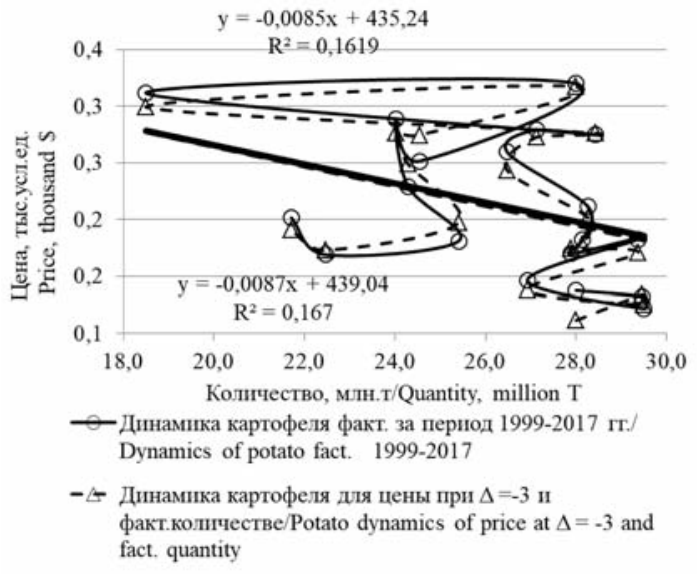

Рис.7. Сопоставление расчетной и фактической цены при фиксированном количестве картофеля (1999-2017 годы) Fig. 7. Comparison of the estimated and actual prices for a fixed amount of potatoes (1999-2017)

ной связи и высокую дисперсию полученных результатов. Однако очевидна обратно пропорциональная связь между количеством и ценой продукта на рынке, так сокращение количества картофеля на 1 млн тонн может привести к росту рыночной цены на 8,5 усл.ед/т (см. значение коэффициента 0,0085 в анализируемой зависимости).

Проанализируем динамику количества (на 31 мая) к цене (на 31 декабря) картофеля (рис.5). При фактических данных имеет место линейная зависимость $\mathrm{y}=$ 0,015x+630.83 при коэффициенте достоверности $\mathrm{R}^{2}=0.3018$. Значение коэффициента достоверности имеет большее значение, чем у предыдущей зависимости. Однако и в этом случае, сокращение количества картофеля на 1 млн тонн может привести к росту рыночной цены на 15 усл.ед/т (см. значение коэффициента 0,015 в анализируемой зависимости).

Рассчитаем значения временного лага при ценовом континууме месячных лагов и константе количества, определим коэффициент корреляции и суммы годовой стоимости картофеля за девятилетний период 19992017 годов для каждого месячного лага (рис.6). Анализ расчетных данных выявляет экстремум (оптимальное время сделки) коэффициента корреляции (наилучшую линеаризацию обратной регрессии) цены/количества) на 31 сентября ( $\Delta=-3)$ текущего периода, т.е. оптимальное время сделки для цены картофеля 31 сентября при количестве на 31 декабря текущего периода (рис.6).

При этом суммарная стоимость (выручка) картофеля за период тем больше, чем больше задержка сделки.
Если текущее количество будет фиксировано на период (допустим, вследствие неурожая картофеля или импортных ограничений), то более позднее совершение сделки формирует большую стоимость (выручку) от реализации картофеля.

Рассматривая соотношение (рис.6) цена/количество картофеля при фактическом и оптимальном варианте, очевидно, что изменение временного лага не имеет существенного влияния на динамику цены. То есть в оптимизации временного лага рынка картофеля определяющее значение имеет количество, а не цена (сопоставление рис. 5 и 7).

\section{Выводы}

Несмотря на ряд концептуальных допущений, предложенная методика определения месячных значений показателей по фактическим годовым представляет практический интерес для управления в условиях неопределенности или недостаточности информации. Апробация методики демонстрирует возможность применения оптимизации временного лага для исследования аграрного рынка. Несмотря на простоту и доступность изложенной методики, имеются ряд вопросов, связанных с погрешностью статистических данных и неучтённых факторов, требующих дальнейшего исследования и научного обсуждения.

В ходе апробация установлено следующее.

1. Выявлено ежегодное сокращение валового сбора картофеля в среднем на 364,6 тыс.т, что составляет $1,2 \ldots 1,7 \%$ от годового вала.

2. Выявлен обратно пропорциональный характер зависимости цены производителя от количества картофеля, т.е., чем хуже урожайность, тем выше цена производителя. Сокращение предложения картофеля на 1 млн тонн на аграрном рынке РФ может привести к росту цены производителя на 8,5 \$/т (4-5\% в среднем).

3. Подтверждена гипотеза о линеаризации равновесных рыночных процессов в условиях временных сдвигов динамики их параметров. Проверено предположение о существование межинтервального временного оптимума, при котором линеаризация бизнес-процессов максимальна.

4. В ходе корреляционного анализа динамики годовых показателей рынка картофеля выявлен месяц май текущего периода как оптимальное время предложений производителя при ограничениях цены и убывающей доходности, и месяц сентябрь - при ограничениях количества и возрастании доходности. При фиксировании цены производителя на агарном рынке более вероятны сделки в мае, при неурожае (ограниченном количестве) - в сентябре.

\section{Об авторе:}

Шабанов Т.Ю. - кандидат экономических наук
About the author:

Timofey Yu. Shabanov - Candidate of Economic Sciences

\section{- Литература}

1. Кильматов Т.Р. Временной лаг как фактор потери устойчивости экономической системы. Экономика и математические методы. 2013;49(3):120-122. 2. Корнилов М.В., Сысоев И.В., Безручко Б.П. Оптимальный подбор параметров прогностических моделей в методах нелинейной причинности по Грейнджеру в приложении к сигналам, характеризуемых хорошо выраженными временными масштабами. Нелинейная динамика. 2014;10(3):279-295. 3. Геворкян Э.А., Мартиросян А.Э. Макроэкономическая модель Калецкого с учетом инвестиционного временного лага. Экономика, статистика и информатика. Вестник УМО. 2015;(4):13-15.

\section{- References}

1. Kilmatov T.R. Time-Lag as a factor of loosing of the economic system. Economics and mathematical methods. 2013;49(3):120-122. (In Russ.) 2. Kornilov M.V., Sysoev I.V., Bezrychko B.P. Optimal selection of parameters of the forecasting models used for the nonlinear Granger causality method in application to the signals with a main time scales. Russian Journal of Nonlinear Dynamics. 2014;10(3):279-295. (In Russ.)

3. Gevorkyan E.A., Martirosyan A.E. Macroeconomic Kalecki's model in view of an investment temporary lag. Statistics and Economics. 2015;(4):13-15. (In Russ.) 